\title{
Social Stigmatization: Effects of Emotional Loneliness and Social Integration
}

\author{
Setareh Kamali ${ }^{\mathrm{a}}$, Elizabeth Darvick ${ }^{\mathrm{a}}$
}

\begin{abstract}
The current study examined the effects of loneliness and social integration on social stigmatizations, in order to understand negative perceptions placed on people. The study was a $2 \times 2$ within-subjects, a factorial design that involved participants to read and rate scenarios. The participants were 16 University of California, Los Angeles student's, 14 females and two males. Loneliness had two levels, lonely or not lonely, operationally defined as satisfaction, or dissatisfaction, with target person's current relationships. Social integration had two levels, high or low, operationally defined as being involved, or not, in schoolrelated activities with peers. The dependent variable was social stigmatization, operationally defined as the ratings of psychological attributes given by the participant. Consistent with our data, there were two significant main effects of social integration and loneliness p's $<.001$. However, no significant interaction was found $\mathrm{p}=.905$.
\end{abstract}

Keywords: loneliness, social integration, perception, social stigmatization

The negative connotation around loneliness has left many lonely people being in a double-blind with fewer opportunities for social growth. The perceived level of emotional loneliness, not being satisfied with interpersonal relationships, often has negative stigmatization on the subject even though they are not socially lonely-having many connections to peers and family. However, if the "emotionally lonely" person has many external social connections, does the perception of them become a more positive one? By acquiring a more thorough understanding of the relationship between perceived emotional loneliness and sociability, we can determine how people, who fit this category, are perceived by others. If the target person was described as being lonely, the initial impression of them would be more negative. Lau and Gruen (1992) studied this hypothesis by manipulating the target person's level of loneliness as well as manipulating the target person's sex in four conditions: lonely male, lonely female, non-lonely female, or non-lonely male. Ninety-six participants, 48 females and 48 males, were asked to read a brief description of each manipulation and then were asked to rate how much they liked the subject. The experimenters provided participants with unique attributions that described the level of loneliness of the target subject. The lonely condition presented the female subject as socially lonely regarding friendships, while the male subject lacked familial relationships. The non-lonely condition presented the female as being well sociable with friends, and the male subject as being well integrated with his family.

Lau and Gruen (1992) found a significant main effect in the target's level of loneliness and no significant main effect of the target's sex nor the participant's sex. There was also a significant interaction between loneliness and target person's sex, such that lonely females were rated to be less attractive than non-lonely females, and the difference between nonlonely and lonely males was of a less magnitude.. This is important to our study because it shows that the emotions connected to perceived loneliness do determine the level of positive perception of the subject. In our current study, we proceeded to use level of loneliness as one condition, however, changed the target's sex to target's level social integration. While perceived satisfaction of relationships may affect the attractiveness, or likability, of a person if the social environment around an emotionally lonely person is well established, would notable negative internal emotions still effect social stigmatization?

Even though the sex of target person is a valid influence to whether or not a friendship is wanted by the participant, we feel it is a weakness to the internal validity of the study because of unknown previous relations with a specific sex by the participant. This could confound how they now perceive all subjects of that sex. For example, if a participant had a very negative experience with someone who is male, it is possible they would not view anybody of that sex the same thereafter. By controlling the sex of the target and providing more information about their internal feelings of loneliness and adding descriptions of their social life, we are able to observe whether their emotional loneliness or social integration is a stronger factor of social stigmatization. Another weakness we found in Lau and Gruen's (1992) study was their definition of loneliness. They defined it as the inability to create social relationships. In the current study, we see these two as completely different aspects of a person's life; a person can emotionally feel lonely but continue to have a large number of social connections.

Agneessens, Waege, and Lievens (2006) focused primarily on the specific types of social support and how these different qualitative aspects affected a person's wellbeing. In the study, Agneessens et al. (2006) randomly selected a sampled 623 Belgians, ages 23 to 75 years, to read a sequence of items in a survey depicting different layers of social support. The purpose of this study was to investigate the different types of social support and in what situations they were perceived as fitting in best. The independent variable was the different social scenario layers presented to the participants. A couple of these social support scenarios included when a person needed someone to talk to, financial support, or companionship when a person wanted to go on a vacation. The study continued by having the participants match up these scenarios with whom they would contact for the specific support needed. After conducting the surveys, Agneessens et al. (2006) presented the results. A few of these results showed us that immediate kin relationships are most valuable in regards to financial social support, and friendships are equal to kin relationships for conversing as well as needing a companion for a vacation. 
The importance of this study is the observation of the different relationships and in what situations these specific relationships serve best for social support. Since the Agneessens et al. (2006) study resulted in specific significant effects of different social relationships and social support, it helped maintain internal validity in our current study when matching up our target person's loneliness to their scenario. By matching a targeted lonely college student with their internal emotions and feelings of inadequate social support, we based the scenarios around the social relationships mentioned based on the results of this study. In the current study, for example, the low socially integrated and lonely target person expresses a want for experiences and conversations with potential friends. By manipulating our scenarios to reflect the results of their study, we strengthened our external validity due to the previously studied relations of social support.

The research conducted in Agneessens et al. (2006) study was mainly in the form of categorization. We found this to be a weakness. While determining what type of social support role fits best with a specific life event, it does not mention how the lack of this support may affect a person who is not receiving it. This is important because it is possible that the interaction between external environment and internal emotions are what impact the perception of lonely people.

Nurmi, Toivonen, Salmela-Aro, and Eronen (1996) conducted a study focusing on the behavioral and cognitive strategies young adults use based on their loneliness. The strategies that were focused on are either approach or avoidance oriented and how that corresponds to optimistic and pessimistic views. The goal of the study was to correspond the way that people deal with uncomfortable situations with their level of social anxiety. It was hypothesized that approach or avoidance oriented methods of interaction determine the perception of target person in terms of their social interaction while choosing optimistic or pessimistic views would determine their level of social anxiety. Each participant had a tutor, and the tutors were instructed to rate subjects' social behavior anxiety and possible avoidance strategies. The results expressed that approach oriented strategies often led young adults to show low social avoidance and excuses (Nurmi et al., 1996).

This is relevant to our current study in terms of a third person rating perceived targets. This study based the targets cognitive behaviors to determining the outcome of their social interactions. In the current study, we manipulated the determination of a social interaction outcome to the raters personal preference to have a social relationship with the target person. This provides us with a real-life scenario in which we could determine if someone would want to pursue an interpersonal relationship with the target person solely based on their internal thoughts versus quantity of previously established social connections. One weakness we found in their study was that tutors may not have substantial enough conversations with their students to determine whether or not they avoid social situations. We built off of this weakness and provided the rate with specific scenarios that operationally define loneliness and social connections in the target person, instead of basing the ratings off of observation.

The current study measured the impact of how lonely a person feels on the inside versus how they appear in social connections and relationships on the outside to how this effects an outsiders perception of the quality of an interpersonal relationship a person offers. Lau and Gruen (1992) helped us determine how the target person's loneliness would be presented to the participant. From this study, we concluded that a written scenario with controlled aspects of loneliness for each manipulation would be most valuable for our research. Continuing with their study, we decided that the sex of the target person was insignificant to our manipulation. Therefore, we chose the top four girl names in 1995 for the names of target persons to control for a possible confounding variable of the gender of the target person.

The research done by Agneessens et al. (2006) shaped our research process with the results they provided. This study used their results in regards to what social scenarios match to what kind of social support needed. By using these previously tested results, we increase our external validity in our results. In the study done by Nurmi et al. (1996), we included the aspect of a third party judging and rating the target persons. For example, participants do not explicitly make observations. Instead, they fill out a survey made by the experimenters. In our study, we controlled what is being rated instead of having the rater observe and determine for themselves.

In our study, we predict a significant main effect of social integration on the perceived social stigmatization of the target person such that a well-integrated person is rated higher than a not well-integrated person. We interpret this as being well integrated socially makes a person more appealing to new friends as opposed to not being well socially integrated. The prediction of this main effect stems from the Nurmi et al. (1996) study, which concluded that the tutor rated well sociable people as more active in searching for new friendships. We also predict a second significant main effect of level of loneliness on the person's social stigmatization and outside perception. The study conducted by Lau and Gruen (1992) lead us to the assumption that lonely people would have a significantly lower rating than the non-lonely people are predicted to have. Since our rating of social stigmatization was operationally defined as the likability of the target person, we predict the lonelier the target person is then the less likable they are by the participant.

Along with two significant main effects, we also predict an interaction between level of loneliness and social integration such that the well-integrated subjects are predicted to be rated higher with the non-lonely condition than the lonely condition. Similarly, the subjects who are not well integrated are predicted to rate higher in the non-lonely condition than in the lonely condition. The not well socially integrated subjects are predicted to have a larger magnitude in the difference between the lonely and non-lonely perceptions. The degree of the first independent variable (social integration) on the dependent variable, depends on the degree of the second independent variable (loneliness). When the target person is perceived as lonely, they will score a little higher in the well-integrated condition than in the not wellintegrated condition, and if they are perceived as non-lonely they will also be rated significantly higher in the wellintegrated condition than the not well-integrated condition. With this prediction, we are able to conclude that it will be likely that the well integrated and non-lonely targets would receive higher scores from our participants, which then corresponds to a much less degree of social stigmatization. 


\section{Method}

Participants

The participants included 16 students $($ Mage $=20.2$ years) enrolled in a psychology research methods course at the University of California, Los Angeles. The participation in this study was mandatory in order to pass the course. There were two males and 14 females participating in this study.

\section{Design}

The experiment was a $2 \times 2$ within-subjects, factorial design. Our first manipulated independent variable was the degree of loneliness. The two levels in this first independent variable were either a high degree of loneliness (lonely) or low degree of loneliness (not lonely). In the lonely condition, the subject was described as lacking deep connections and understanding of their peers and family. In the non-lonely condition, their emotional connection to friends and family was high, along with the ability to connect with people when a problem arises. The second manipulated independent variable was social integration. The two levels of this independent variable included a high level of social integration and a low level of social integration. The high level of social integration was operationally defined as having a large network of social connections in school, and the low level was defined as lacking many social connections in school. The dependent variable was social stigmatization, which was measured on a five-point interval scale. We operationally defined that as the ratings of psychological attributes such as adjustment, sociability, achievement, and interpersonal interaction given by the participant, which mirrored the scales used by the Lau and Gruen (2006) study.

\section{Materials and Apparatus}

In our study, each participant received a unique packet made up of nine pages stapled together. These nine pages included one page of instructions and four pages of one descriptive paragraph. After each one paragraph page followed a double-sided page with 24 survey questions that were identical after each paragraph page (e.g., first manipulation paragraph page, double-sided survey page, second manipulation paragraph page, double-sided survey page). This was done so that the participants did not know what they were looking for while reading the first description. The survey questions were identical after every paragraph and were held constant between all 16 participants. The first page included instructions that gave participants directions on their task. This explained to the participant that they would read a description paragraph, of a fictional character, and at the end of each paragraph they would fill out 24 survey questions on the next page provided.

The paragraphs described four girls: Jessica, Ashley, Emily, and Samantha. We used the most common names in the year 1995 in order to hide the manipulation and reason for the study from the participants. The reason for these names was that the participants in this study were all born near the year 1995. Each fictional character was matched to every four conditions using a balanced Latin square. The four conditions were: (a) well socially integrated and lonely, (b) well socially integrated and not lonely, (c) not well socially integrated and lonely, and (d) not well socially integrated and not lonely. Each paragraph included three sentences describing the level of loneliness, and four sentences describing the level of social integration. In order to be consistent with our participant group, each paragraph described the character as a college student. The details of the paragraph were changed depending on which condition they were in. A template paragraph was made for each of the four girls and was then manipulated depending on their condition.

We operationally defined social integration as (a) involvement in campus organizations - being involved or not being involved, (b) living situation-living with roommates or living alone, and (c) the number of peer relationships they made - made many friendships or not has not made many friendships. We operationally defined loneliness to be the level of satisfaction with connections with one's peers and family-either satisfied or dissatisfied and desires better relationships. When creating the four character descriptions, the three sentences that described loneliness contained information about personal satisfaction with their relationships as well as included how they would respond in times of stress.

The four sentences that described social integration focused on each aspect of the operational definition given. In regards to loneliness, we used words such as "misunderstand" and "reluctantly" in o order to embody a character who was not emotionally comfortable in the current situation. In contrast, in the non-lonely condition, we included "honored" and "belonging" that showed a sense of pride and comfort in her situation. The character's stories were novel, however still included their given condition (e.g., well socially integrated/lonely). An example of a template social integration sentence is: "Ashley [lives near the school with her best friends]/[commutes to her university and lives alone]." A loneliness sentence template was for example: "During her free time at school, [she spends time with her classmates]/[she studies in the library]." The first option of the sentence was always the positive (well socially integrated/not lonely) one and the second option was the negative one (not well socially integrated/lonely). Depending on the balanced Latin square, we matched the given name's story with the appropriate condition. There was no particular order in which the independent variable conditions were presented in the paragraph so that the description was easy to follow.

The page that followed each individual paragraph included 24 surveys, each ranging from 1 (most negative) and 5 (most positive). These surveys included questions that rated the participant's perception of the character (e.g., "how much do you like this person," and "would you be friends with this person"). The remaining survey questions also stemmed from the Lau and Gerald (1992) study and asked participants to include subjects' assessment on a scale of 1-5 (e.g., weakstrong, ugly-attractive, and lonely-not lonely). A lower score indicated the more negative connotations of the target person.

\section{Procedure}

Participants were randomly assigned to one of the 16 packets. They were instructed not to begin until the experimenter told them to do so. The experimenter then read the instruction page out loud to the participants and asked if there were any questions before proceeding and they were instructed to indicate their age and gender on the instruction page. The participants were instructed to flip the page and begin reading their first scenario after the instructions were 
read and there were no questions. They were instructed to fill out the survey questions after reading each paragraph in consecutive order. They were then told to flip the page and read the paragraph then answer the surveys on the following pages as the experimenter started the stopwatch. The participants were given 18 minutes to read all four scenarios and answer the surveys that followed each page. After the participants finished, the experimenter proceeded to collect all the packets in no particular order. Once all the packets were collected, the experimenter then asked if the participants had any questions regarding the experiment.

\section{Results}

Figure 1 displays the average stigmatization scores that were given by participants that present not lonely versus lonely as a function of the level of social integration. After analyzing the graph, it seems that there was a more positive score if the passage described a non-lonely person rather than a lonely person. On average, there was also more positive scoring on people that were portrayed as well socially integrated than if they were portrayed as not well socially integrated.

In order for these effects to be tested, we analyzed the data using a two-way, within-subjects ANOVA. The results presented a significant main effect of loneliness, such that the average stigmatization score was significantly higher when the subject was perceived as not lonely $(\mathrm{M}=3.90, \mathrm{SD}=0.37)$ than if they were perceived as lonely $(M=2.91, S D=0.31)$, regardless of the level of social integration of the subject, $\mathrm{F}(1,15)=91.34, M S E=0.17, \mathrm{p}<.001$. A significant main effect of social integration was also revealed, such that the average stigmatization score was significantly higher in the well integrated subjects $(\mathrm{M}=3.66, \mathrm{SD}=0.37)$, than when they were presented as not well socially integrated $(\mathrm{M}=3.15$, $\mathrm{SD}=0.34)$ regardless of the level of loneliness, $\mathrm{F}(1,15)=$ 20.62, MSE $=0.21, \mathrm{p}<.001$. The interaction depicted in Figure 1 was revealed to be non significant, $\mathrm{F}(1,15)=.015$, $M S E=0.14, \mathrm{p}=.905$.

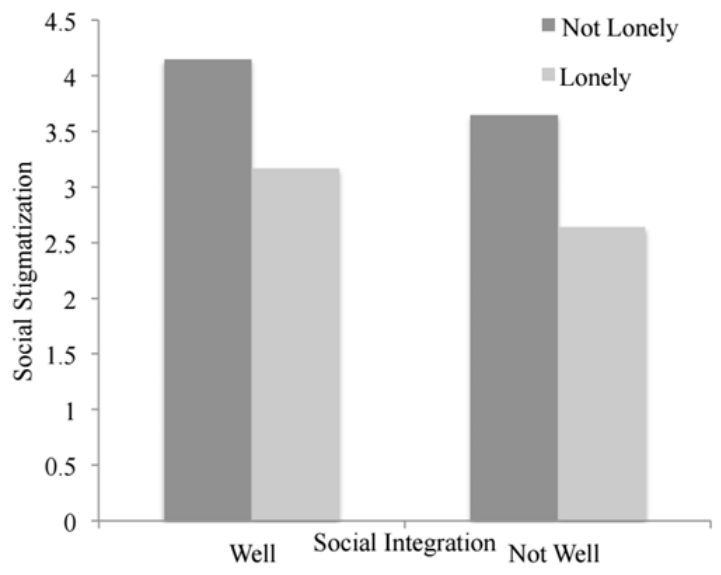

Figure 1. Average social stigmatization scores with the manipulation of social integration and loneliness. Well socially integrated was scored significantly higher than not well socially integrated, regardless of loneliness, and not lonely scored significantly higher than lonely regardless of social integration. No significant interactions were found.

In order for us to compare individual means, multiple ttests, with a Bonferroni correction in order to keep an alpha level of .05 , were performed. When the fictional character was presented as not lonely, the average score given by the participant was significantly higher when the subject was well socially integrated $(M=4.15, S D=0.56)$, than when they were not well socially integrated $(\mathrm{M}=3.65, \mathrm{SD}=0.41), \mathrm{t}(15)$ $=3.13, \mathrm{p}<.0125$. When the subject was perceived as lonely, the average score given was also significantly higher when the subject was well socially integrated $(\mathrm{M}=3.17, \mathrm{SD}=$ $0.45)$, than when they were not well socially integrated $(\mathrm{M}=$ $2.64, \mathrm{SD}=0.35), \mathrm{t}(15)=4.03, \mathrm{p}<.012$. Therefore, as Figure 1 depicted, level of loneliness had a significant effect on social stigmatization regardless of when subjects were presented as well socially integrated or not well socially integrated.

When the subjects were portrayed as being well socially integrated, the average score given to them was significantly higher when they were paired with being not lonely ( $\mathrm{M}=$ $4.15, \mathrm{SD}=0.56)$, than when they were also perceived as lonely $(\mathrm{M}=3.17, \mathrm{SD}=0.45), \mathrm{t}(15)=5.64, \quad \mathrm{p}<.012$. Similarly, when the subjects were presented as not well socially integrated, the average score given to them was also significantly higher when they were not lonely $(\mathrm{M}=3.65$, SD $=0.41)$, than when they were seen as lonely $(\mathrm{M}=2.64, \mathrm{SD}=$ $0.35), \mathrm{t}(15)=10.90, \mathrm{p}<.012$.

\section{Discussion}

The current study concluded that there was a significant main effect of social integration on social stigmatization, such that subject's were rated less negatively stigmatized if they were well socially integrated than if they were not well socially integrated with their surroundings. There was also a significant main effect of loneliness, such that when people were perceived as being non-lonely, they scored, on average, higher than when they if they were in the lonely condition. Both of these significant results were consistent with our hypothesis. However, there was not a significant interaction between social integration and loneliness. This was inconsistent with our hypothesis because we believed that subjects would be rated fairly similar in the well socially integrated condition regardless of loneliness, and very different in the not well socially integrated group based on the level of loneliness.

Lau and Gruen (1992) found a significant main effect of loneliness, which indicated that loneliness was a significantly higher stigmatized state than non-loneliness. This was consistent with our current study results. Based on their adjustability scales, a lonely person is resulted to be less achieving, rated weaker, and more passive than a non-lonely person. We also found this to be consistent in our current study. What this shows us is that when somebody is viewed as being dissatisfied with a few, if not all, the relationships in their life, they are often stigmatized to a much more negative level. One possible problem may have been that our levels of loneliness were not well concealed, possible making our study's intent apparent. This may have influenced the participants to give the answer they believed we were testing. The operational definition of loneliness in the study by Lau 
and Gruen (1992) was being incapable of making social ties with surroundings. We believed that it was appropriate to define that as social integration and shift loneliness to an internal emotion. This study differed in the way that loneliness was operationally defined. Changing the definition to a more internalized state of mind was important because while there are many forms of loneliness, emotional loneliness, in our opinion, was one that would make the most impact on social stigmatization.

Agneesens et al. (2006) study found that there was a significant main effect on the type of social support one receives. They concluded that for different situations in life, such as a financial crisis, going on vacation, or needing someone to talk to, different relationships are more fitting to help a person through that specific situation. Social integration, and external relationship factors, also resulted in a significant main effect in the current study. We hypothesized that there would be a significant main effect of social integration, such that well socially integrated people would on average be rated higher (less stigmatized) than not well socially integrated people. We hypothesized this because we believed that people's social environment would be a significant factor in the level of stigmatization. The current study differed from the Agneesens et al. (2006) study because we focused primarily on whether or not any social interactions exist, as opposed to the types of interactions and their benefit. This was done because we believed the absence of social interactions as a whole would make a much greater effect on social stigmatization, than simply manipulating the interaction would. By doing this, we also decreased the external validity due to the fact that many people simply do not lack social integration as a whole. The variability in the Agneesens et al. (2006) study made it more externally valid. Therefore, our study did not generalize to the population as a whole, instead focuses on specifically very well socially integrated people and people who are not socially integrated at all.

Lau and Gruen (1992) also found significant interactions between loneliness and sex of target person, such that lonely females were rated to be less attractive than non-lonely females, and the differences between non-lonely and lonely males were of a less magnitude. We hypothesized that if the sex of the target person is an influencing factor, with loneliness, to alter the stigmatization, then social integration should not be less of an influence. Since a person's sex is not often changeable, and social integration is, we believed that social integration would be more of a stigmatizing factor, being that it is under the control of the target person. However, in the current study, we did not observe a significant interaction between loneliness and social integration. The difference in the findings could be due to the fact that the Lau and Gruen (1992) study was focusing on the sex of target person, and their participants had an equal amount of male and female participants. However, in our study, we could not balance the second variable (social integration) between our participants. For example, if half of our participants were well socially integrated themselves, and half were not, then we may possibly have seen a significant interaction. Since we had a group of students who were well socially integrated, even just in the classroom environment itself, this could have acted as a bias.
The current study had a few limitations that reduced the external validity. With the design of the current study, we can conclude that people who are not socially integrated at all are often perceived more negatively than people who are very involved in their college campus. This study cannot conclude that overall social integration and loneliness have negative overall stigmatizations on people. We can only conclude that college students will often stigmatize people who are very lonely more than people who are not lonely at all. This limits our external validity and ability to tie connections to the general population. Another limitation of the study was that the participants read a passage that ultimately described feelings of discontent that the fictional subject's felt. This would not happen outside of an experiment; people are not aware of the thoughts and emotions of others regarding the satisfaction of their relationships unless it is vocalized. By giving the participants information that would not be given on a day to day basis about a subject, they were able to understand them on a much deeper level, a level that would not exist just by looking at someone on campus. This limits our ability to understand how social integration and loneliness dependently effect stigmatization.

Future studies may build off of the current one to improve many aspects of it. The first thing that would increase the external validity of the study would be to manipulate the loneliness variable on a visual scale. In this instance, people would look at pictures of subjects who seem to have characteristics of a lonely person instead of reading a description of what thoughts are going through their head. This would increase the external validity because in the real world this is how people first perceive someone without interacting with them. In the current study, social integration was defined as the number of social connections, living situation and the involvement in school activities. Future studies could build off of this and test whether or not the popularity of the subject affects their level of stigmatization. If someone is deemed to be very well-liked, and have many friends, would that be more positively viewed than if they are very well-liked, but have a few friends? What would be the observed outcome of somebody who is not popular (not wellliked) but still is well socially integrated? Testing quality over quantity would be interesting when applying it to social perceptions and would help us begin to understand the motivation that some people have to be very well-liked by a lot of people, and also what factors (popularity or social integration) plays a more important role in positive perceptions.

The current study observed evidence for two main effects, one of loneliness on social stigmatization, and the other of social integration on social stigmatization. Regardless of the many external limitations of the study, the results are useful in breaking the previous stereotypes of loneliness and social integration. It is a common misconception that if somebody is not well socially integrated with their peers, they are also very lonely. This leads to an array of misunderstandings of a person. The opposite statement is also true. Simply because somebody is perceived to have many friends, and being very involved on campus, does not conclude that they are not feeling emotionally lonely. Whether somebody is friends with a lot of people or is involved with school campus activities, or not, does not determine their internal emotions. After these, many times 
false, assumptions are made the attitudes and behaviors towards that person changes. Everybody should get the chance to be understood before having positive or negative perceptions put on them. By understanding and researching what causes highly negative perceptions, we can begin to inform and educate the population in these differences, in hopes of decreasing future negative stereotypes that ultimately affect how we initially treat others.

\section{References}

Agneessens, F., Waege, H., \& Lievens, J. (2006). Diversity in social support by role relations: A typology. Social Networks, 28 (4), 427-441.

doi:10.1016/j.socnet.2005.10.001

Lau, S., \& Gruen, G. E. (1992). The social stigma of loneliness: Effect of target person's and perceiver's sex. Personality and Social Psychology Bulletin, 18 (2), 182-189. doi: 10.1177/0146167292182009

Nurmi, J., Toivonen, S., Salmela-Aro, K., \& Eronen, S. (1996). Optimistic, approach-oriented, and avoidance strategies in social situations: Three studies on loneliness and peer relationships. European Journal of Personality, 10 (3), 201-219. doi:10.1002/(SICI)1099-

0984(199609)10:3<201::AID-PER257>3.0.CO;2-\# 